\title{
Biografía, historia e identidad: una propuesta y un ejemplo
}

\author{
AURELIA VALERO PIE
}

El que la biografía no sólo debe examinar las acciones y el contexto en que se desarrolló la vida del personaje biografiado, sino también los postulados que sostienen la concepción misma del sujeto, constituye la tesis central de estas páginas. Según algunos análisis de Norbert Elias, el artículo se centra, a título de ejemplo, en José Gaos y su proyecto autobiográfico. Identificar el trasfondo psicoanalítico en su escritura tiene por objeto señalar que su personalidad no corresponde del todo al modelo forjado durante el siglo $\mathrm{XIX}$, periodo en el que los rasgos del individuo liberal se erigieron como paradigma de la naturaleza humana. Desprenderse de ese modelo para reconstruir las estructuras de sentido que sostienen cada identidad sería, por ende, una competencia del género biográfico.

Palabras Clave: identidad, Norbert Elias, José Gaos, Sigmund Freud, psicoanálisis, autoanálisis

\section{Biography, History, and Identity: A Proposal and an Example}

This article argues that biographies should not only examine the context, work and deeds of biographees, but also their own conceptions of identity. Following the analysis of Norbert Elias, it takes as an example José Gaos' autobiographical project. The purpose of identifying the psychoanalytical background that lies beneath Gaos' writing is to show that his personality does not correspond to the model fabricated during the 19th century. It was then when the idea and attributes of the liberal individual was erected into a paradigm of human nature. To question that model and to reconstruct the structures of meaning which sustain each identity would be, in that sense, a competence of the biographical genre.

Aurelia VAlero Pie

El Colegio de México,

Programa de Investigadores Asociados,

Distrito Federal, México

aureliavalero@gmail.com
KEYWORDS: identity, Norbert Elias, José Gaos, Sigmund Freud, psychoanalysis, self-analysis 
n 1989, hace un cuarto de siglo, Jacques Le Goff describía aquellos años como una "época de retornos": al individuo, al acontecimiento, a la biografía (Le Goff, 1993). De ese modo señalaba las novedades que se habían manifestado en la producción historiadora durante los últimos tiempos y que apuntaban hacia una transformación del modelo historiográfico hasta entonces imperante. El que la biografía, considerada durante largos años como una narrativa menor, cuando no indigna de la investidura académica, figurara entre las formas reconocidas de la disciplina era testimonio de la magnitud de los cambios operados. De la historia de la literatura hasta la otra que se ocupa de la ciencia, pasando por las también recién resurgidas corrientes política e intelectual, el género cumplía la proeza de mantener su popularidad entre el público lector — sobre todo en Europa-, al tiempo que ganaba el aprecio de los profesionales de la disciplina.

El renacimiento de la biografía marca, si no el final del paradigma estructuralista y de su concomitante ideal cientificista, al menos el declive de la hegemonía que éstos ejercieron durante varias décadas. Uno y otro ofrecieron sustento a numerosas investigaciones en historia social, a cuya luz el género aparecía como un producto falsamente historiográfico, carente de valor en términos tanto metodológicos como teóricos. Ambas deficiencias explican que entre los historiadores sociales se le considerara como un adorno con el que la literatura infestaba de bisutería la historia. De ahí que en el medio académico francés se le reputara, a lo sumo, como una "modesta herramienta que ayuda a observar o a ilustrar mejor las tendencias de largo aliento, las estructuras y las gravitaciones; si bien en modo alguno puede pretender erigirse en una palanca intelectual" (Bonin, citado en Loriga, 2010: 211). No menos acerbas fueron las críticas proferidas en Alemania, donde se juzgó al género como el último reducto del historicismo alemán, dado que en él se expresaba nada menos que el "frecuentemente dogmático principio de individuación historicista” (Oelkers, citado en Bödeker, 2003: 12). La concepción del sujeto que se plasmó en la biografía aparece, en consecuencia, como responsable en gran medida del carácter anacrónico e incluso retardatario que durante casi medio siglo se prestó 
—al menos en ciertas vertientes y tradiciones historiográficas - al género en su conjunto. ${ }^{1}$

Apenas sorprende, por lo demás, el que la biografía quedara asociada al paradigma individualista de impronta liberal, en vista de que se trata, como bien observó Norbert Elias, de un fenómeno común a las ciencias humanas y sociales, al menos como se desarrollaron durante gran parte de la centuria pasada. De manera implícita, unas y otras basaban sus análisis en las formas de emotividad, comportamiento y experiencia contemporáneas, sin advertir que cada tiempo y espacio posee particularidades propias. De ahí que en el trasfondo de los conocimientos producidos se encontrara el homo clausus, es decir, "la imagen del ser humano aislado, como un ser completamente libre y completamente independiente, como una 'personalidad cerrada', que depende de sí mismo en su 'interior' y que está separado de los demás individuos" (Elias, 2012: 56). No eran inocuas las repercusiones de esa idea para la sociología y su capacidad de explicación, dado que entre postular una supuesta autonomía universal del sujeto y disociar individuo y sociedad sólo había un paso que, una vez franqueado, imposibilitaba dar testimonio del cambio social. El lugar de esa disciplina en la cartografía del saber quedaba de este modo invalidado. Una situación muy distinta aparecía, sin embargo, cuando se prestaba una respuesta diferente al "endiablado problema de la conexión entre las estructuras psicológicas individuales, esto es, de las llamadas estructuras de personalidad, y las composiciones que constituyen muchos individuos interdependientes, esto es, las estructuras sociales" (Elias, 2012: 33). Dicha respuesta consistía en considerar ambos planos, no sólo desde el punto de vista de su condición histórica, sino "como aspectos interdependientes del mismo desarrollo a largo plazo" (Elias, 2012: 33).

Mediante un estudio detallado de ciertos hábitos y costumbres cultivados en la Europa moderna, Elias invitó a comprender la identidad como un movimiento continuo, a la vez índice y factor de las transformaciones verificadas en el ámbito colectivo. También resaltó que en el surgimiento del yo y en los procesos de subjetivación el lenguaje desempeña un papel determinante, mientras que, al revisar los conceptos "de causalidad psíquica y de causalidad social", puso "en relieve el lugar del cuerpo, las operaciones de vínculo con los otros y consigo mismo" (Mier, 2008: 137). Ahora bien, de ser verdad que la biografía busca reconstruir no sólo el contexto y las acciones del individuo biografiado sino el horizonte que les brinda sentido, las consecuencias tanto en la práctica como en la teoría no pueden desatenderse. Ellas apuntan, en efecto, a la necesidad de conocer, junto con los actos y las circunstancias que marcaron el desarrollo de una vida en específico, los modelos, categorías y conceptos que en cada caso ofrecen sustento y contenido a cierta personalidad. Las concepciones de la realidad, las proyecciones temporales y las redes de vínculos, fundadas en signos, discursos y hábitos, se desplazan con ello a un primer plano. No podría ser de otra forma, puesto que, de olvidar

Cabe destacar que los casos francés y alemán no agotan el espectro de apreciaciones que han rodeado a la biografía. En Gran Bretaña, por mencionar un ejemplo eminente, el género ha gozado de una tradición ininterrumpida y sólida, mientras en otros países el paulatino abandono se verificó de un modo a lo sumo parcial. Ello responde, sin duda, como señaló uno de los dictaminadores de este artículo, al potencial que guarda este tipo de narrativa para alcanzar el éxito en las ventas y difundirse así entre sectores relativamente amplios de la sociedad. Explicar su permanencia depende, por lo mismo, de una mirada más atenta a la historia de la lectura y del libro como empresa cultural. De este fenómeno es prueba el medio mexicano, donde el alejamiento nunca fue completo: mientras el relativo declive de la biografía parece haberse producido sólo en el espacio académico a raíz de la llamada "profesionalización" de la enseñanza, la recepción de obras como las de Enrique Krauze muestran que el género nunca perdió del todo su lugar entre el público lector. Para conocer una evaluación temprana de los estudios elaborados en esta vertiente, véase O'Gorman (1945). 
ese conjunto de factores, se corre el riesgo de difuminar el carácter histórico que distingue toda identidad, así como las estructuras que la hacen posible y le van prestando un significado. Al desestimar o dar por sentado los elementos inscritos en los procesos sociales de individuación, los acercamientos de tipo biográfico perderían, al menos en potencia, una parte de su función y fundamento, es decir, la de entender las claves de una existencia y los caminos por los que el individuo biografiado pudo llegar a ser.

No han faltado, desde luego, quienes han cuestionado el modelo basado en el sujeto liberal al momento de emprender un estudio de naturaleza biográfica. Un buen ejemplo aparece en el que en años recientes François Dosse dedicó a Paul Ricoeur, quien se encuentra moldeado conforme a la idea que este último arbitraba acerca de la identidad personal. Se entiende así que el libro adoptara una forma dialógica y que el filósofo objeto de análisis apareciera "a partir de la mirada múltiple de los otros, del entrecruzamiento de itinerarios y de encuentros sucesivos" (Dosse, 2013: 19). Asimismo, resultó imperativo abandonar aquel modelo para quienes han buscado biografiar personalidades oriundas de coordenadas distintas a las que comprende el mundo occidental. Así lo expresó R. Keith Schoppa en sus reflexiones en torno a la figura de Shen Dingyi, líder comunista chino que destacó en las primeras décadas del siglo Xx. Al advertir que las redes en las que este personaje se desenvolvía aportaban mayores herramientas para esclarecer su trayectoria que los rasgos intrínsecos a su personalidad, mostraba la necesidad de desprenderse de ciertas concepciones implícitas en el canon biográfico moderno. Entre ellas se contaban nociones como la "satisfacción personal" —individual fulfillment— y el desarrollo del propio potencial — self-realization-, es decir, ideas derivadas del paradigma liberal decimonónico y su "glorificación de lo individual" (Schoppa, 2004: 28). Conocer la vida y la trayectoria de un espectro más amplio de actores dependía, por lo mismo, de la habilidad para renovar las premisas en las que por lo común descansa el género biográfico.

Una propuesta análoga se expone en el presente artículo, al tomar como ejemplo a José Gaos, filósofo exiliado en México a raíz del derrocamiento de la Segunda República española. Si su caso resulta ilustrativo, se debe a que el homo philosophicus ${ }^{2}$ parece encarnar en grado sumo los rasgos del tipo de individuo que se han discutido en estas líneas. Más aún, en la medida en que su concepción del sujeto cimienta no sólo gran parte de su obra, sino las formas de experiencia disponibles en ese contexto particular, dilucidar los engranajes que sostienen su identidad resulta imprescindible para cualquier reconstrucción biográfica que lo elija como objeto. De otro modo sería incomprensible la tenacidad con la que a lo largo de sus días exploró los ejes de su conciencia y personalidad, centrales en su pensamiento filosófico, y sobre todo en la necesidad de fundamentar una de sus principales tesis, a saber, que pese a su apariencia abstracta y ambiciones de absoluto, toda filosofía no es sino una confesión personal. A ello se debe el que aportar pruebas relativas al origen individual de las ideas fuera el cometido que persiguió con mayor ahínco y que explica también la infatigable reflexión que consagró a su propia actividad intelectual. Así se entiende que entre sus escritos, tanto publicados como inéditos, figuren profusos pormenores relativos a sus vivencias, lecturas y recuerdos, registrados con tanta minuciosidad que con frecuencia aparecen con la fecha inscrita al margen.

Se trata de la figura que Norbert Elias, con base en la tradición, describió como "el individuo aislado [que] consigue conocimiento sobre el mundo 'fuera de él mismo' y por sus propios medios", aquel que "no necesita aprender de los demás" (Elias, 2012: 56). De Descartes a Husserl, la razón serviría, según este modelo, como único sustento de ideas y representaciones; se olvida que en unas y otras subyacen los procesos de socialización en los que toda persona se halla inserta desde la niñez y a lo largo de la edad adulta. 
Quienes conozcan la historia del género autobiográfico habrán identificado tanto en el planteamiento del proyecto como en su modo de proceder los rasgos de una doble corriente que desde el siglo XIX impregna, en palabras de René Girard,

el pensamiento y la sensibilidad de la época: por un lado, la exaltación del sentimiento y la moda de las confesiones, siguiendo las huellas de Rousseau; por otro, la ambición de los ideólogos de fundar la ciencia del hombre sobre la observación, colocando la sensación en el origen del entendimiento, de acuerdo con Locke, Helvétius y Condillac (Girard, 1996: 32).

Limitarse a invocar los referentes clásicos de la tradición, sin examinar las premisas concretas que le prestan sustento, implicaría, sin embargo, reducir el proyecto de Gaos a una expresión tardía del romanticismo burgués, retocado con tintes ilustrados. No se comprendería el carácter eminentemente moderno de sus escritos ni las razones por las que fracasó en el intento de poner en blanco y negro los pormenores de su existencia, en tanto clave explicativa de sus ideas y personalidad. Tanto su vida como su obra permanecerían, por ende, en una relativa oscuridad.

Una imagen muy distinta aparece al advertir que, entre los modelos recurridos, se cuenta también el psicoanálisis y numerosas nociones forjadas en la psicología que se desarrolló con el cambio de siglo. De ahí que examinar el papel que desempeñó el método que fundó Sigmund Freud en la escritura autobiográfica de Gaos, así como en el desarrollo de su pensamiento filosófico, constituya el propósito

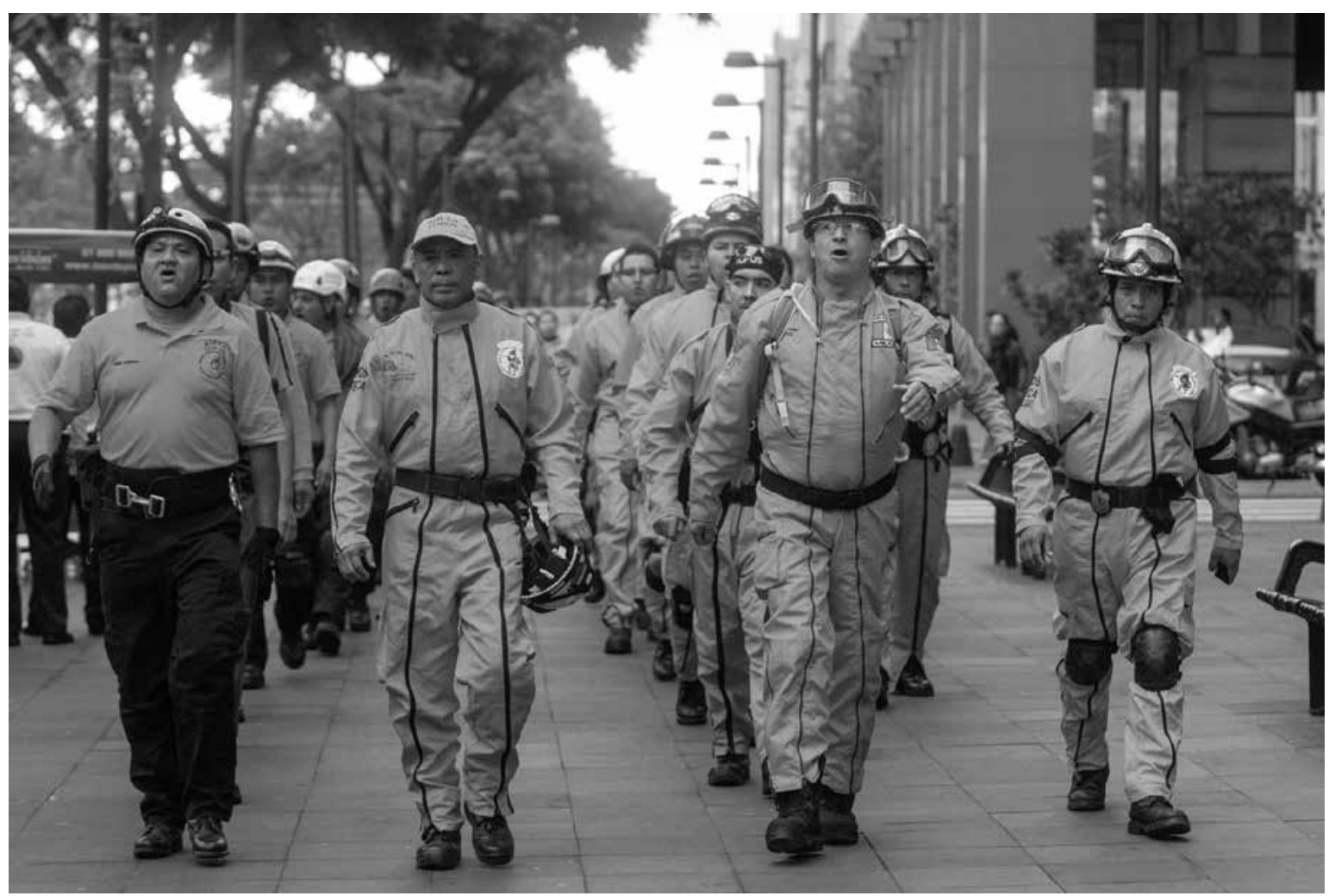

Rodrigo GonzÁlez • Topos marchan por avenida Juárez en el aniversario 28 del sismo de 1985. Ciudad de México, 19 de septiembre de 2013. 
de las páginas siguientes, divididas en tres secciones. En la primera expongo los principales argumentos de la polémica que el filósofo "transterrado" sostuvo con Francisco Larroyo, de modo que aparezca la centralidad del psicoanálisis para el desarrollo de su doctrina. En el segundo apartado busco mostrar el lugar de la escritura en la construcción del yo y señalar los conceptos y mecanismos de origen freudiano que Gaos empleó para dar testimonio de su propia identidad. A modo de cierre, apunto hacia la intervención de estructuras de carácter social —en este caso, representadas en los principios psicoanalíticos - en la conformación de la intimidad, para sugerir la complejidad, historicidad y polivalencia que entraña la noción misma de sujeto biográfico.

\section{Filosofía y psicología en discordia}

Si bien la concepción de la filosofía como confesión personal se remonta a los años de José Gaos en España, la necesidad de fundamentar esa idea mediante conocimiento de orden psicológico y procedimientos psicoanalíticos parece haberse hecho evidente hasta su llegada a México, cuando Francisco Larroyo decidió poner a prueba los conceptos del visitante español e instigó la reflexión del desafiado en torno a los mecanismos y posibilidades de la introspección. La polémica comenzó durante las conferencias que Gaos pronunció entre el 17 y el 22 de octubre de 1938 en el paraninfo de la Universidad Nacional. ${ }^{3}$ Eran sus presentaciones inaugurales en la capital y la intriga del público —alimentada por las abundantes noticias que la prensa difundía sobre su figura - se expresó en la nutrida concurrencia que colmó la sala. Se trató, según se dijo, de "todo un acontecimiento", en razón de que "estaban muy lejanas las ocasiones en que habíamos escuchado a otros conferenciantes extranjeros, sobre todo, acerca de un problema filosófico" (Menéndez, 1940: 4). No obstante, si algo logró congregar a "señoras y políticos, profesores y estudiantes", no fue tanto el atractivo de escuchar aquellas elevadas disquisiciones, cuanto

una curiosidad natural de aquel momento, producida por la inmigración española de tipo intelectual, [...] determinada, en algunos, [por] una cierta compasión por unos señores que acababan de perder a su patria, o bien, en otros, por una antipatía basada en razones de credos sociales y políticos. No faltó como incentivo, en cierta clase de público, un despecho nacionalista, es decir, de monroísmo mexicano (Menéndez, 1940: 4).

Asistido por su consumado arte oratorio, el recién llegado logró conducir a ese heterogéneo grupo de oyentes por los oscuros senderos de su "filosofía de la filosofía", título del ciclo en su conjunto.

Según explicó en la primera intervención, "filosofía de la filosofía" constituía un sintagma de origen diltheyano que denotaba el acto de estudiar la disciplina en sus propios términos. Tal era, precisó, el sentido de la reflexión que hacía todo filósofo al tomar por objeto de estudio, no tanto a Dios, el Mundo y el Hombre, cuanto a la naturaleza del saber que enmarcaba y orientaba esa misma reflexión. Dicho de otro modo, se trataba de la búsqueda, tan antigua como la propia filosofía, de su definición. Lejos de propugnar con ello algún tipo de nominalismo, el expositor invitaba a indagar sobre su significado a partir de manifestaciones efectivas en el seno de la existencia. Con base en ese postulado, explicó al auditorio, su alocución sólo versaría sobre la manera en que se vivía la filosofía en tanto forma de experiencia, es decir, no como un conocimiento pretérito, sino como una expresión del presente

Los pormenores y el alcance de la polémica se han desarrollado en Valero (2012: 124-147). Retomo aquí unos cuantos pasajes. 
y no desde el punto de vista del objeto, sino del sujeto. La referencia a la subjetividad en modo alguno se perdería en vagas abstracciones. Por el contrario, tan concretos serían sus razonamientos que en realidad constituirían "confesiones", dado que "hablar de filosofía es siempre hablar de sí mismo”. De ahí que agregara: "no os dejéis engañar bajo los términos generales; yo voy a hablar fundamentalmente de mí mismo. Mas no sólo de mí mismo [...], porque hablar de sí mismo es siempre hablar un poco de los demás" (Gaos y Larroyo, 2003: 69).

En ese espíritu distinguió cuatro etapas en la relación con la disciplina cuya cuna se encuentra en las costas del Mediterráneo: vocación, profesión, decepción y obstinación, en el entendido de que con ellas expondría "la historia, el cuento y también la confesión de esta vocación, de esta profesión, de esta decepción, de esta obstinación en el orden cronológico que es el natural de las cosas" (Gaos y Larroyo, 2003: 69). Aquellas fases, deducidas de su propia experiencia, concentraban lo que denominó una "prosopopeya del filósofo", es decir, una descripción de la personalidad que distinguía a los miembros de su gremio. Esa personalidad se revelaba por una especie de llamado que se designa, tanto en la religión como en el lenguaje corriente, con el término de vocación. Quienes a él respondían no eran individuos cualesquiera, sino los marcados por un signo que no distaba mucho del que, según las leyendas gnósticas, empujó hacia el éxodo al primer hijo de Adán. En el vocabulario de Gaos, ese signo se denominaba "afán de saber", un impulso irresistible por conocer los principios que rigen al mundo y a sus habitantes. ¿Qué mejor medio para satisfacer ese deseo que una disciplina que predicaba poseer, como un remedo del fruto prohibido, el conocimiento primero, último y definitivo? Atraído por la promesa, el elegido decidía consagrar su pensamiento y existencia al cultivo de la filosofía y hacer de ella una profesión. Pero al igual que en el relato bíblico, después de la transgresión viene la caída, el ávido de saber termina por descubrir que se trata de un voto vacío y que la tan ansiada verdad universal y absoluta es, en realidad, relativa y perecedera. Llegaba entonces la decepción, momento en que el estudioso comprendía que sus esfuerzos habían sido en vano y que sus palabras representaban meros sonidos que se llevaba el viento. Pero ya era demasiado tarde: dado que la filosofía no es ciencia de los primeros principios, saber definitivo ni verdad absoluta, queda abierta la pregunta sobre qué era aquello que había informado su vida. El filósofo comenzaba a interrogarse sobre la naturaleza, sentido y función de la disciplina, persistía en el empeño de filosofar. Por consiguiente, de la obstinación surgía de forma casi inevitable una "filosofía de la filosofía”. Con ella se cerraba el ciclo y quizá también las puertas del Paraíso.

En la prosopopeya descrita hay un elemento que se ha reservado para el final, debido a su significación, tanto en la doctrina de Gaos como en la polémica a que dio lugar. Se trata de la soberbia, rasgo que consideraba como el más característico entre sus pares, pasados y contemporáneos. Del latín super-eia, "estar encima”, “dominar”, ese atributo revelaba la voluntad de poder subyacente en la vocación por la filosofía y el "afán de saber", en la profesión y la consagración de la vida a su cultivo, en la decepción y el apetito siempre insatisfecho, y sobre todo, en la obstinación y su persistencia en ella. Términos casi intercambiables, filosofía y soberbia mantenían entre sí una "armonía preestablecida", dado que "en ambas — sostuvo - se dan las mismas notas capitales" (Gaos y Larroyo, 2003: 61). Con este paralelismo o asimilación, surge el verdadero sentido de la prosopopeya que, del griego prosopôn, "máscara", significa, justamente, desenmascarar. Ahora bien, arrancar máscaras y tapujos constituía el objetivo que acometió, al reconocer que el origen del filosofar radicaba, más que en una búsqueda desinteresada de saber, en un deseo irresistible de dominación. De esa forma, ingresaba en lo que Paul Ricoeur denominó "escuela de la sospecha": la que sostiene que 


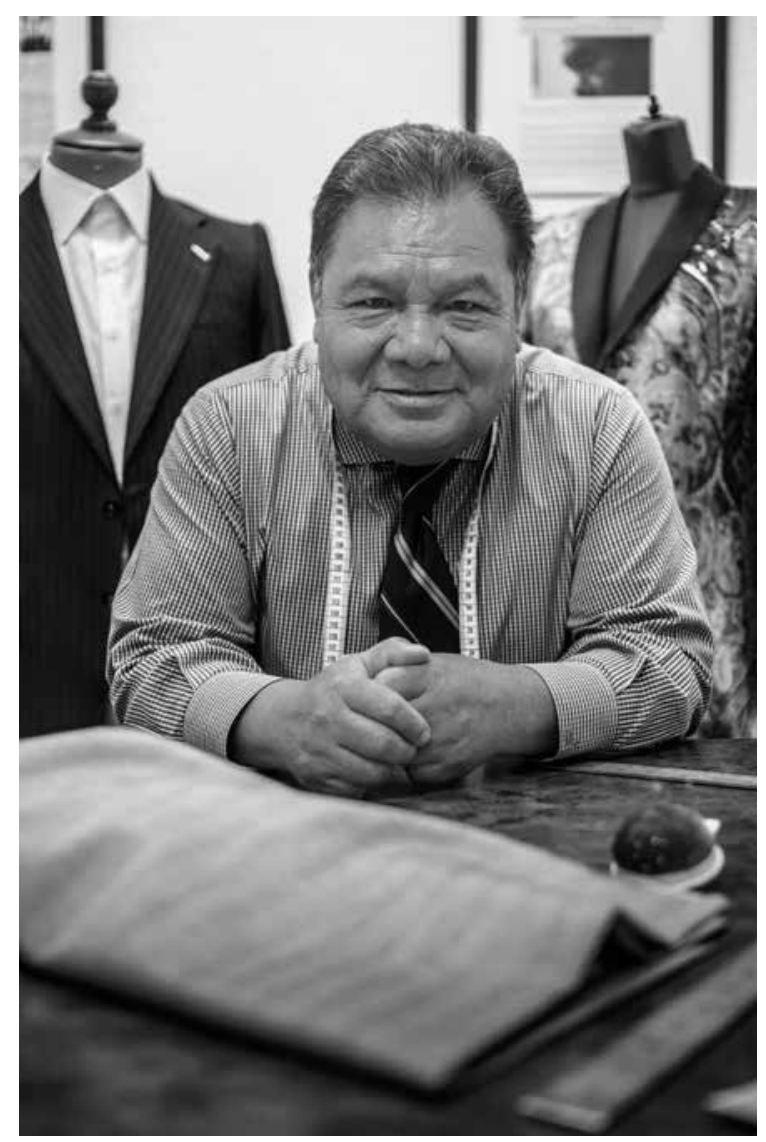

RODRIGo GonZÁLEZ • Gilberto, reconocido como el mejor sastre del mundo por la casa de telas Scabal. Ciudad de México, abril de 2015.

nuestra percepción se confunde en apariencias, sólo disipadas mediante la observación y el análisis atentos (Ricoeur, 1965: 42-46). ${ }^{4}$ A partir del modelo de los grandes maestros - Karl Marx con el concepto de ideología, Friedrich Nietzsche con la noción de resentimiento y Sigmund Freud con el descubrimiento del inconsciente-, Gaos encontró una realidad filosófica marcada por el ánimo de superioridad. Sin embargo, como el ángel caído, el soberbio estaría condenado a la frustración terrena. A ello se debía, concluyó, que "el filósofo es el hombre que muestra al hombre sus límites, su finitud, y ejerce así una función regulativa de la existencia humana" (Gaos y Larroyo, 2003: 63).
Al decir de la prensa, las conferencias gozaron de un gran éxito entre el público de la capital, así como entre algunos miembros reconocidos del medio cultural, en particular Antonio Caso. Pese al temprano éxito, no faltaron, sin embargo, quienes disintieron de los postulados y definiciones que propuso el recién llegado. Entre ellos destaca Francisco Larroyo, un joven profesor de filosofía convertido al neokantismo, en la vertiente axiológica de la Escuela de Baden, tras estudiar en las universidades de Friburgo y Heidelberg. Si bien fueron numerosas y muy afiladas las objeciones que entonces profirió en las páginas de la revista Hoy, sólo se mencionarán aquellas que recusaban las etapas del filósofo y el concepto de soberbia en tanto pretendida esencia del filosofar. Bastaba con evocar el ágape de Platón, la vida contemplativa en Santo Tomás o el voto de Descartes de peregrinar a Loreto para comprobar que no era tal el impulso de la meditación filosófica. Pero si los testimonios históricos no convencían del todo, menos aún lo hacía la metodología del conferenciante. Al menos así lo pensó el detractor, para quien las "reflexiones en torno a la actitud subjetiva del hombre que filosofa podría intitularse la psicología del filósofo". A todo ello agregaba incisivo que por mucho o poco valor que encerrara "la historia o cuento de las vivencias personales del profesor Gaos [...], no deja de ser un análisis empírico de su conciencia a muchas leguas de distancia del método filosófico de la reflexión trascendental" (Gaos y Larroyo, 2003: 66-70). De esa forma quedaba desmantelada, en varios de sus puntos cardinales, la doctrina del huésped de ultramar.

Quizá inseguro de cómo actuar en el medio que lo recibía, o con mayor probabilidad, a causa de una arraigada aversión por la controversia y la

4

Tan buen alumno resultó Gaos de esa escuela, que en años posteriores se propuso realizar un "autopsicoanálisis marxista y nietzscheano”. AJG, 4, exp. 4, f. 62242, 13 de enero de 1959.

Biografía, historia e identidad: una propuesta y un ejemplo 
publicidad, el desafiado emitió su respuesta no en los canales públicos de la prensa, sino mediante una carta dirigida al autoproclamado polemista. En ella argumentaba que la documentación histórica no rebatía la idea de un "afán de dominación" en tanto motor del filosofar, puesto que esos ejemplos representaban "la máscara de la soberbia del soberbio ‘débil'” (Gaos y Larroyo, 2003: 74-75). Por último, rechazaba que su recurso remitiera a una mera psicología e invocaba a su favor el amparo del propio fundador de la fenomenología:

La filosofía de la filosofía comprende una psicología del filósofo. Pero esta psicología, no puede hacerse sino mediante conceptos que son objeto de fenomenología. Así la fenomenología de la soberbia es indispensable a la psicología del filósofo en cuanto soberbio. Pero hay, además, una fenomenología de la filosofía y del filósofo. Esta fenomenología, como toda fenomenología, debe hacerse a base de casos ejemplares empíricos y puede hacerse a base de uno solo e incluso imaginario o ficticio, enseñanzas todas expresas de Husserl (Gaos y Larroyo, 2003: 76).

Para júbilo de los editores, "embelesados" ante la posibilidad de "promover y sostener un diálogo impulsado por el afán de verdad”, Gaos atendió “a la súplica de algunos profesionales de México”, al consentir que la carta se publicara en la misma revista que había acogido la primera reseña. ${ }^{5}$ Ésta venía acompañada de una nueva y profunda estocada por parte de quien había llamado a duelo, llegaba al corazón de la doctrina rival y también al fondo de la polémica: "Averiguar, psicológicamente —escribió Larroyo- , si el hombre que filosofa cae en el sentimiento de la soberbia, no puede hacerse en última instancia, sino por el método de introspección (auto-observación)" (Gaos y Larroyo, 2003: 84). En vista del carácter singular e intransferible de dicho procedimiento, no era posible comprobar que el atributo aducido constituyera el resorte de los esfuerzos por descifrar el mundo con el alfabeto filosófico. Para redondear su argumento, el crítico añadió: "Comparto con el profesor Gaos que la confesión personal sea una esencia captable fenomenológicamente; pero de que la fenomenología pueda descubrir la esencia de confesión personal no se sigue de ningún modo que la filosofía se caracterice con semejante esencia" (Gaos y Larroyo, 2003: 84-85). Palabras más, palabras menos, en la contrarréplica del así aludido se sostenía que las diferencias remitían a dos concepciones distintas de la filosofía, con lo cual se llegó a un callejón sin salida y se dio por concluida la controversia.

\section{Una construcción del yo en clave psicoanalítica}

En 1940, con los auspicios de La Casa de España en México, apareció Dos ideas de la filosofía (pro y contra la filosofía de la filosofía), libro que enmarcó el diálogo entre uno y otro filósofo. En el prólogo del volumen se sostenía que el motivo de la publicación radicaba en su carácter "instructivo" y como ejemplo de la "veraz cooperación entre la intelectualidad de España y la de la América española” (Gaos y Larroyo, 2003: 47). No cabe duda de que ambos atributos resultaban valederos para al menos uno de los polemistas. Aunque en su correspondencia juzgó la postura de su interlocutor como "más que nada, curiosa", las notas privadas de Gaos muestran que no desestimó las impugnaciones recibidas. ${ }^{6}$ En efecto, si el propósito estribaba en demostrar el origen biográfico de toda filosofía, en tanto prueba y fundamento de su naturaleza "confesional", parecía imprescindible superar un escollo. Se trataba de la dificultad que muy acertadamente percibió Larroyo

$5 \quad$ Hoy, año II, vol. VII, núm. 95, 17 de diciembre de 1938, p. 36.

6 Carta de José Gaos a Francisco Romero, 20 de enero de 1940 (Torchia, 1992). 
y que radicaba en encontrar un punto de intersección entre filosofía y psicología. De hallar una salida dependía nada menos que su doctrina entera, puesto que el método elegido consistía en estudiar su propia personalidad para comprender a sus colegas, y de ahí, el ramo del saber que entre todos cultivaban.

El primer y más evidente problema era el de la inducción, es decir, el acto de inferir reglas generales a partir de un solo caso. ¿Resultaba lícito atribuir experiencias similares a quienes, como él, habían hecho de la filosofía una profesión? La pregunta, relativa a los vínculos entre el individuo y la sociedad, no tardó en hallar una respuesta acorde con unos fines dispuestos de antemano: "En rigor —se convenció-, no separo la vocación de mi vocación: ésta queda subsumida en aquélla". ${ }^{7}$ Así se lo mostraba la fenomenología contemporánea, cuyas generalizaciones permitían ir absorbiendo los ejemplos examinados, incluso sin importar si se trataba de uno solo. De sus lecturas en psicología extrajo enseñanzas análogas: "En realidad — reflexionó en una nota-, mi individualización del tipo es el lugar y la clave de las otras... aunque o precisamente porque mi individualización del tipo es obra de las otras: en cuanto informantes de mi individualización han tenido su lugar y clave en ésta”. El corolario le pareció tan evidente como inevitable: "la autobiografía es el lugar de la historia y de la tipología", ${ }^{8}$ concluyó en un argumento que lo convirtió, por obra de aquel frágil silogismo, en exemplar y exemplum del homo philosophicus en general.

Una vez zanjada esa cuestión, quedaba por establecer las operaciones que desvelarían el origen común y subjetivo de la disciplina, con lo cual se desmontarían, además, algunas críticas de Francisco Larroyo. Al meditar sobre la naturaleza de esa rama del saber, determinó que no se hallaba desligada de sus manifestaciones concretas en el tiempo y el espacio: "Pero esta Historia — advirtió en seguidatiene una previa heurística: la que en mí, para mí, ha sido, es, la filosofia". El punto de vista del sujeto quedaba de esta forma restablecido, con la ventaja de que lograba equiparar mediante esas premisas la filosofía y el autoanálisis. Un salto argumentativo más fue suficiente para elegir y validar el procedimiento que guiaría el experimento:

La Psicología contemporánea ha venido a ser también autoanálisis, como método preferente, por más generalizable, de una higiene mental. Filosofía y Psicología convergen. La Psicología llegaría a Filosofía, si el autoanálisis fuera suficientemente radical hasta los principios. La Filosofía debe usar los métodos de la Psicología también, si no exclusivamente. ${ }^{9}$

Únicamente a partir de ese cruce disciplinario podría alcanzarse la finalidad terapéutica, el examen histórico y una heurística de la filosofía.

El autoanálisis, punto de arranque hacia una nueva teoría de la mente, constituye el gesto inaugural, pero también irrepetible e inimitable del psicoanálisis como ciencia autónoma. Así lo han asentado quienes buscan practicar las enseñanzas de Sigmund Freud y ello sin obstar que el origen de descubrimientos sorprendentes, como el significado de los sueños y el complejo de Edipo, se hallara en las

7 AJG, 1, exp. 101, f. 20293, 29 de marzo de 1940. [Cursivas en el original.]

8 AJG, 1, exp. 101, f. 20291, 29 de marzo de 1940. [Cursivas en el original.]

9 AJG, 2, exp. 33, ff. 35695-35696, 4 de mayo de 1944. En otra nota apuntó: "Filosofía es para mi higiénico autoanálisis histórico, radica/ y llevado, o traído, hasta concluir que él, y ella, no es suficiente higiénicamente. La Filosofía encuentra su método en la Psicología. Pero ¿será posible descubrir por el método de la asociación de ideas las raíces inmanentes-trascendentes de nuestra vida? La Psicología ha venido a ser (auto)análisis sociológico-histórico -insuficiente, no radical- que debe radicalizarse, esto es, hacerse Filosofía. La Psicología encuentra su perfección en la Filosofía". AJG, 2, exp. 33, f. 35703. [Cursivas en el original.] 


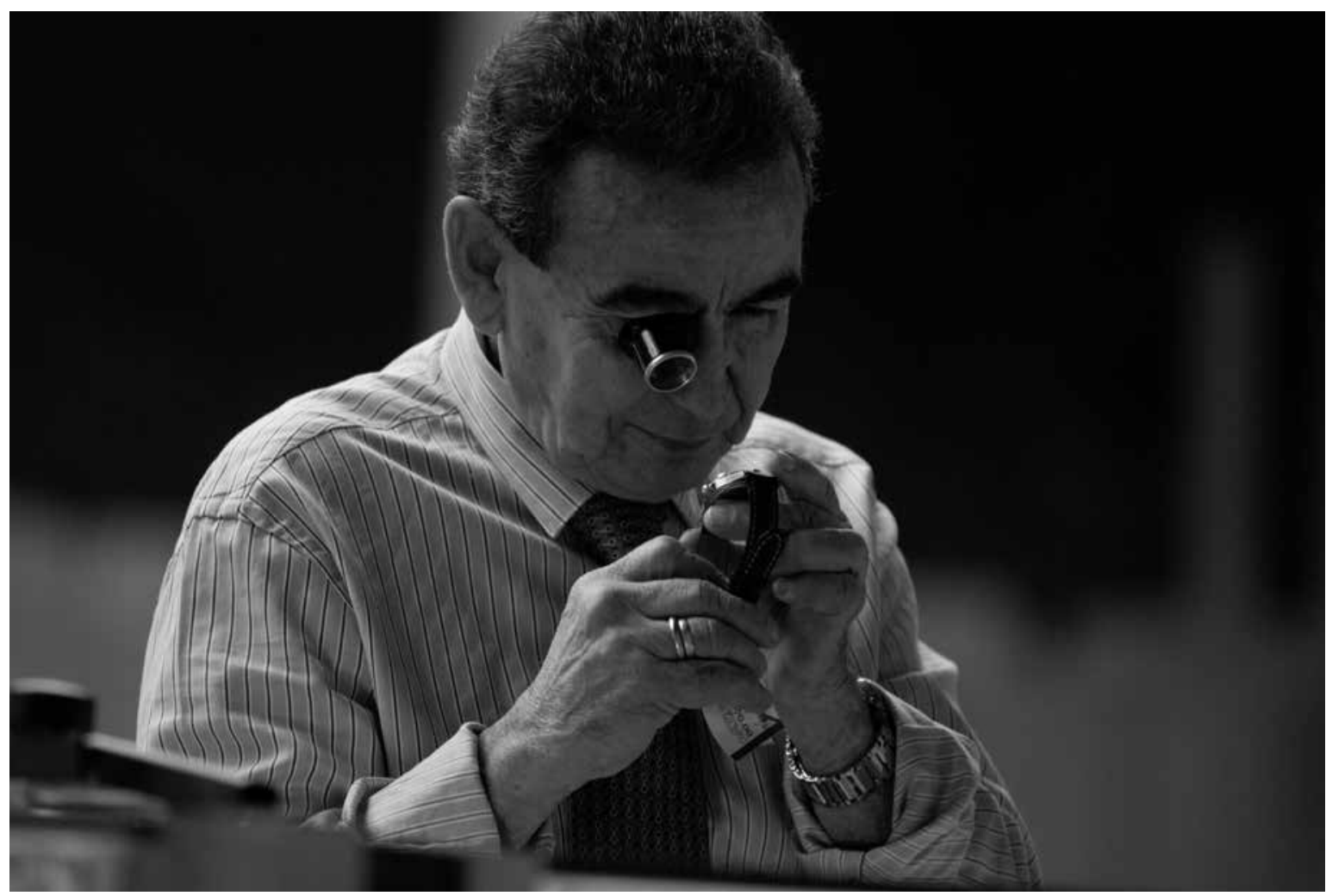

Octavio Hoyos • Trabajador del Monte de Piedad. Ciudad de México, julio de 2014.

exploraciones de su propia conciencia. ${ }^{10} \mathrm{El}$ mismo fundador de esa disciplina defendió con insistencia la prioridad de la mirada ajena y llegó a reconocer que "el verdadero autoanálisis es imposible". "Sólo puedo analizarme —explicó a Wilhelm Fliess en noviembre de 1897- mediante un conocimiento logrado objetivamente (como un extraño)" (citado en Gay, 2010: 125). Nada de ello impidió que en las primeras décadas del siglo anterior el autoanálisis representara un método muy recurrido entre sus discípulos y lectores en distintas partes del planeta, incluyendo a aquellos que se encontraban en tierras mexicanas y en la península ibérica (Gallo, 2014; Saiz y Saiz, 1996).

Según estudiosos del movimiento, hacia finales de la década de 1920, "el psicoanálisis en España había pasado a ser un conjunto de hipótesis y técnicas incorporadas eclécticamente a la teoría y a la praxis por la mayor parte de los psiquiatras, y bastante bien acogido como un sistema psicológico complementario a la visión biologicista" (Muñoz González, citado en Vera, 1996: 429). A difundir ese conjunto de nociones había contribuido José Ortega y Gasset, quien, desde 1917, recomendó a José Ruiz-Castillo, director de la Biblioteca Nueva, que su editorial tradujera las Obras completas de Freud. El origen de la iniciativa no dejará de producir cierto asombro, sobre todo si se considera que no hacía largo tiempo

10

Se ha afirmado, por ejemplo, que "ver en el psicoanálisis una variedad de la introspección equivale a desconocerlo. El conocimiento de sí y la introspección son incapaces de asegurar las bases de una teoría del psicoanálisis, de sus fines, principios y eficacia” (Lagache, citado en Anzieu, 1978: 15). 
que el filósofo madrileño había definido el método psicoanalítico como una "técnica de la purgación o Katharsis espiritual”, semejante, en ese sentido, a una "justificación científica del confesionario". ${ }^{11}$ También juzgó la teoría del inconsciente como un absurdo conceptual, mientras que equiparó los resultados obtenidos con un "chascarrillo" que invita "decididamente a la risa" (Ortega y Gasset, 2004: 489-497). Es de suponer que la progresiva aceptación de las ideas que entonces despreció hiciera mella en su ánimo, dado que en 1922, al aparecer el primer volumen vertido al castellano, alabó las ideas allí impresas como "la creación más original y sugestiva que en los últimos veinte años ha cruzado el horizonte de la psiquiatría". El entusiasmo que denotaban sus palabras no estaba, sin embargo, desprovisto de reservas.

Lo más problemático en la obra de Freud es, a la vez, lo más provechoso. Me refiero a la atención central que dedica a los fenómenos de la sexualidad [...]. Freud amplía notablemente el concepto de la sexualidad que suele llamar libido, pero aun así, ¿no deja su obra siempre la inquietud de que se nos invita a aceptar una hipótesis desmesurada? (Ortega y Gasset, 2005: 410).

Apenas resulta arriesgado imaginar que alguno de los 15000 ejemplares vendidos hasta 1936 (Sánchez, 1996: 350) llegara a las manos de José Gaos ni que las ideas de su maestro le fueran en modo alguno desconocidas. Sin poder determinar cuán familiarizado estaba con el pensamiento de Freud, parece al menos indudable que para 1940, cuando decidió establecer por escrito sus vivencias y recuerdos, el psicoanálisis le prestó herramientas invaluables. ${ }^{12}$ Así lo muestra el que, con el propósito de esclarecer los pormenores de su autobiografía en tanto ejemplo de la vida filosófica en general, por ese entonces se entregara al autoanálisis y a la rememoración. Ajenas al mandamiento de la contratransferencia, las páginas de un diario que comenzó por aquel tiempo conservan el puntual registro de las sesiones en las que él mismo desempeñó los papeles de médico y paciente. Bajo la mirada inclemente de su propia conciencia, día con día se esmeró por puntualizar los matices de su pensamiento, reconocer los rasgos de su carácter e identificar los ejes organizadores de su experiencia vital. El esfuerzo bien valía la pena, dado que al final de ese proceso contaba con descubrir los secretos enterrados bajo la espesura de la piel, y por extensión, los que escondían los miembros de su gremio.

Aunque desprovisto del rigor que reclaman sus mismos practicantes, el psicoanálisis se prestó así a un empleo heterodoxo y a los más variados fines. Entre éstos se contaba la posibilidad de validar la prosopopeya del filósofo - impugnada en su momento por Larroyo - mediante la precisión y la solidez atribuidas a la ciencia. A partir de las herramientas, procedimientos y criterios de la llamada talking cure, la subjetividad del individuo quedaría enmarcada en la objetividad, cualidad indispensable para hacer del temperamento propio un saber universal, o dicho de otro modo, para comulgar "en la

A ese respecto parecía coincidir José Gaos. En una nota titulada "Confesión y psicoanálisis", estableció la siguiente analogía: "el interés era el de un examen de conciencia perfecto y se lograba: por eso no había neurosis. El psicoanálisis puede ser no más que un sustitutivo imperfecto de todo ello". AJG, 4, exp. 2, f. 60601, 2 de noviembre de 1943.

12 Aunque relativamente numerosas, las referencias que Gaos hizo al psicoanálisis a finales de la década de 1930 y principios de la siguiente no me permiten establecer con certeza la profundidad de su conocimiento sobre la materia ni saber si era directo o indirecto. Sólo me es posible afirmar que para 1967, fecha en que redactó unas lecciones sobre Freud, se hallaba plenamente familiarizado con El origen y desarrollo del psicoanálisis, La introducción general al psicoanálisis y el Epitome del psicoanálisis. Otras alusiones, insertas en ese mismo curso, inducen a pensar que al lado de estas obras también había leído, por lo menos, La interpretación de los sueños, Más allá del principio del placer y Tótem y tabú (Gaos, 1994: 513-529). 
verdad de las personalidades". ${ }^{13}$ A fin de cuentas, sostenía Gaos, "el hombre, por naturaleza — necesidad o gusto- conoce y se ha esforzado por conocer científicamente". Ahora bien, continuó, "entre los conocimientos del hombre que se ha esforzado por convertir en científico cuenta el que cada uno tiene de los demás y de sí mismo. Tal es el origen y el concepto de la Psicología”. ${ }^{14}$ Había más. En ocasiones de menor comedimiento, llegó a atribuir a los descubrimientos de Freud el poder de penetrar y transparentar toda conciencia.

Pienso que el psicoanálisis acaba de enseñarme que la Psicología no puede tener por objetivo exclusivamente el potenciar en ciencia la psicología de la vida cotidiana, sino el llegar a saber todo lo posible de lo psíquico ajeno y propio: hay conocimientos psicológicos que no parecen alcanzables por aquella mera potenciación, sino sólo por técnicas exclusivamente científicas [...].

Más directamente importante para mi obra inmediata es el haberme hecho darme cuenta de que mi autobiografía filosófica, en cuanto autognosis, debiera auxiliarse de toda la ciencia psicológica posible. Claro que sólo de aquella que da a conocer realmente a uno, como a los demás, no de la que sólo da a conocer "generalidades fenomenológicas" [...]. Mi Psicología del filósofo debe usar el psicoanálisis del mismo. ${ }^{15}$

Armado con las nociones de esa escuela, enriquecidas con otras más elaboradas desde la corriente de Alfred Adler, se abocó entonces a escudriñar los recovecos de su propia mente e identidad. Dedujo entonces que su "personalidad era, sigue siendo, reducida a lo esencial, la de un individuo de fuerte impulsividad sexual, pero introvertido e intelectual y soberbio". La conjunción de esos rasgos lo había llamado a sublevarse contra el severo catolicismo inculcado en la niñez y en la primera juventud. Según dedujo al hurgar en su interior, en el cultivo de la razón pura habría encontrado una salida a unas estrictas normas de conducta, pero también el medio de reconvertir y satisfacer los imperativos del deseo, fuera éste de índole carnal o intelectual: "Este complejo [...] encontró la pieza con que ensamblaba punto por punto en la filosofía". ${ }^{16}$ Sus pulsiones, animadas por el principio del placer, convergían de nuevo con los atributos de la disciplina.

También su memoria fue objeto de un examen profundo. Si su carácter había servido como un campo de exploración y como un revelador de la soberbia, faltaba averiguar, en efecto, cómo había encontrado un cauce en la vocación filosófica. Con ese fin, el autoanálisis confluyó con un ejercicio de rememoración en el que se esforzó por recordar las sucesivas escalas que marcaron su camino hacia la filosofía. Siguiendo a su manera las enseñanzas del psiquiatra vienés, no tardó en persuadirse de que “el mejor método de rememorar acabadamente el pasado de uno, es el de proceder por asociación de los recuerdos a los tiempos y a los lugares. Es como voy a proceder" ${ }^{17}$ Cientos de apretadas páginas, inéditas hasta hoy, dan testimonio de la seriedad con la que acometió dicha tarea. En ellas intentó precisar, con rigor y sistema, las condiciones de su formación, desde los días de su infancia y hasta su ingreso en la Facultad de Filosofía y Letras de Madrid. Su devoción juvenil, manifestada en el fervor con el que se encomendaba a Dios y asistía a ceremonias de tipo religioso, quedó también consignada en su diario, así como sus excursiones de verano y la imagen que le transmitieron unos cuantos maestros. Fiel a la doctrina, en ningún momento olvidó escrutar los movimientos pasionales y el pulso

\footnotetext{
13 AJG, 1, exp. 42, f. 7297.

14 AJG, 1, exp. 42B, f. 7461, 3 de marzo de 1944.

15 AJG, 1, exp. 42B, f. 7515, 7 de octubre de 1943.

16 AJG, 1, exp. 42B, f. 7423, 8 de diciembre de 1943.

17 AJG, 2, exp. 38, f. 36264.
} 
libidinal, como al recordar las palpitaciones del primer amor y su fijación por las mucamas. El sello que el psicoanálisis imprimió al siglo Xx, con su énfasis en el erotismo y la sexualidad, se hacía así presente en este novel surcador de la conciencia. ${ }^{18}$

Pertenecientes a otro orden de experiencia, las obras que informaron su itinerario intelectual constituyeron asimismo un motivo de reminiscencias. El detalle con el que enumeró títulos y autores, en el orden en que la lectura había tenido lugar, respondía a la necesidad de hacer de su recorrido un periplo común a sus contemporáneos. No era todo: en la medida en que su formación comprendía el saber acumulado durante la era moderna, su trayectoria podría condensar un compendio de la filosofía universal. El vértigo de las listas, como Umberto Eco denominó a esa obsesión, se apoderó de José Gaos, quien no cesaba de componer y recomponer el inventario de los pensadores que habían dejado alguna huella en sus ideas (Eco, 2009). De ahí que los nombres de Taine, Schopenhauer, Nietzsche, Windelband, Husserl, Külpe, Messer, Heidegger y Dilthey, por mencionar unos cuantos, se sucedieran como en una especie de ensalmo espiritista. ¿Qué efectos habían marcado en su espíritu? ¿Cuáles sentimientos le habían producido entonces? ¿Había un acceso no mediado a la experiencia originaria? ¿Cómo abstraer el conocimiento adquirido en años posteriores? Tales fueron algunas preguntas que le despertó ese arduo ejercicio de recuperación.

La descripción de la filosofía y como la encontré yo —reflexionaba Gaos para sí- requiere la historia de mis lecturas y estudios filosóficos iniciales y el recuerdo del aspecto con que la filosofía se me presentó en ellos. Este recuerdo no me da detalles que advierto hoy. Esto plantea el problema de la existencia en el pasado de lo advertido en él en el presente. ${ }^{19}$

No terminaban ahí las dificultades encontradas. Al dirigir los ojos del recuerdo al paisaje del ayer, su

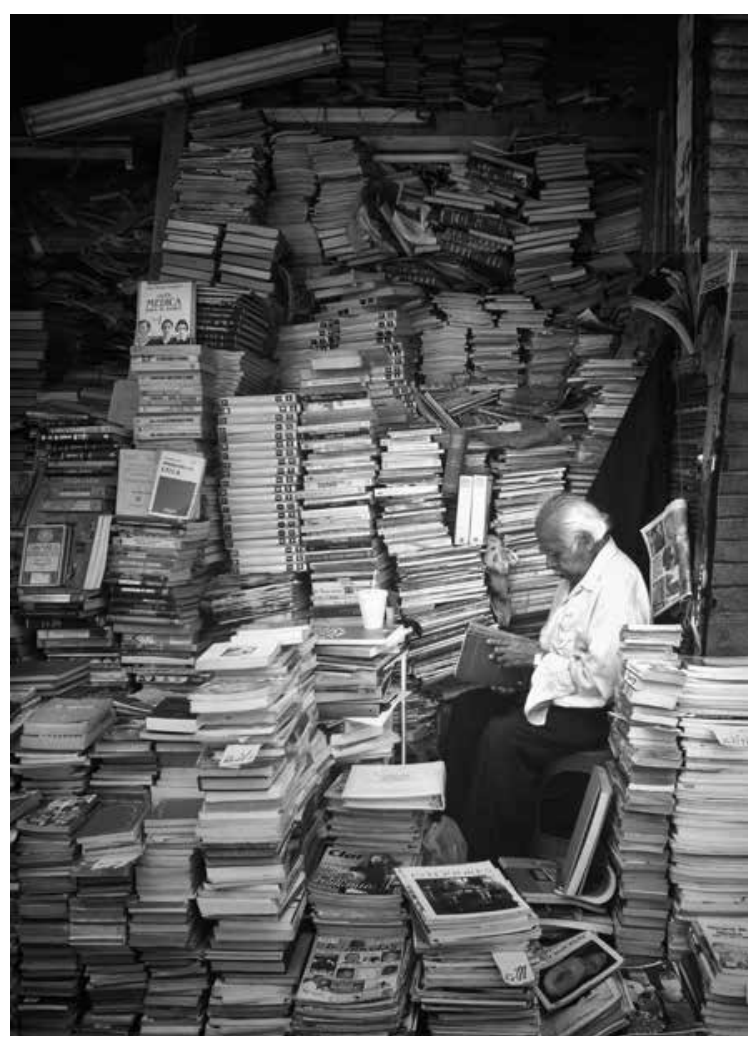

RODRIGO GONZÁLEZ • El librero acomoda su mercancía al final de una jornada en el local que ha ocupado durante más de 20 años. Ciudad de México, abril de 2012.

mirada tropezó con una densa niebla apenas penetrada por efímeros rayos de luz. Un episodio en particular, central en su autobiografía, aparecía envuelto en unas sombras tan espesas que ningún esfuerzo de memoria alcanzaba a esclarecer, o al menos, no con la nitidez que requería su anhelado proyecto. Se trataba del momento de su encuentro con la filosofía, germen de su vocación y punto de arranque de toda su trayectoria. No tardó en reconocer que ese momento se situaba en la primavera

"El psicoanálisis acaba conformando a la moral", advirtió Gaos en una nota suelta, lo que confirma de este modo la impronta que él mismo reconoció a Sigmund Freud. AJG, 1, exp. 38B, f. 6439.

19 AJG, 1, exp. 42, f. 7205. 


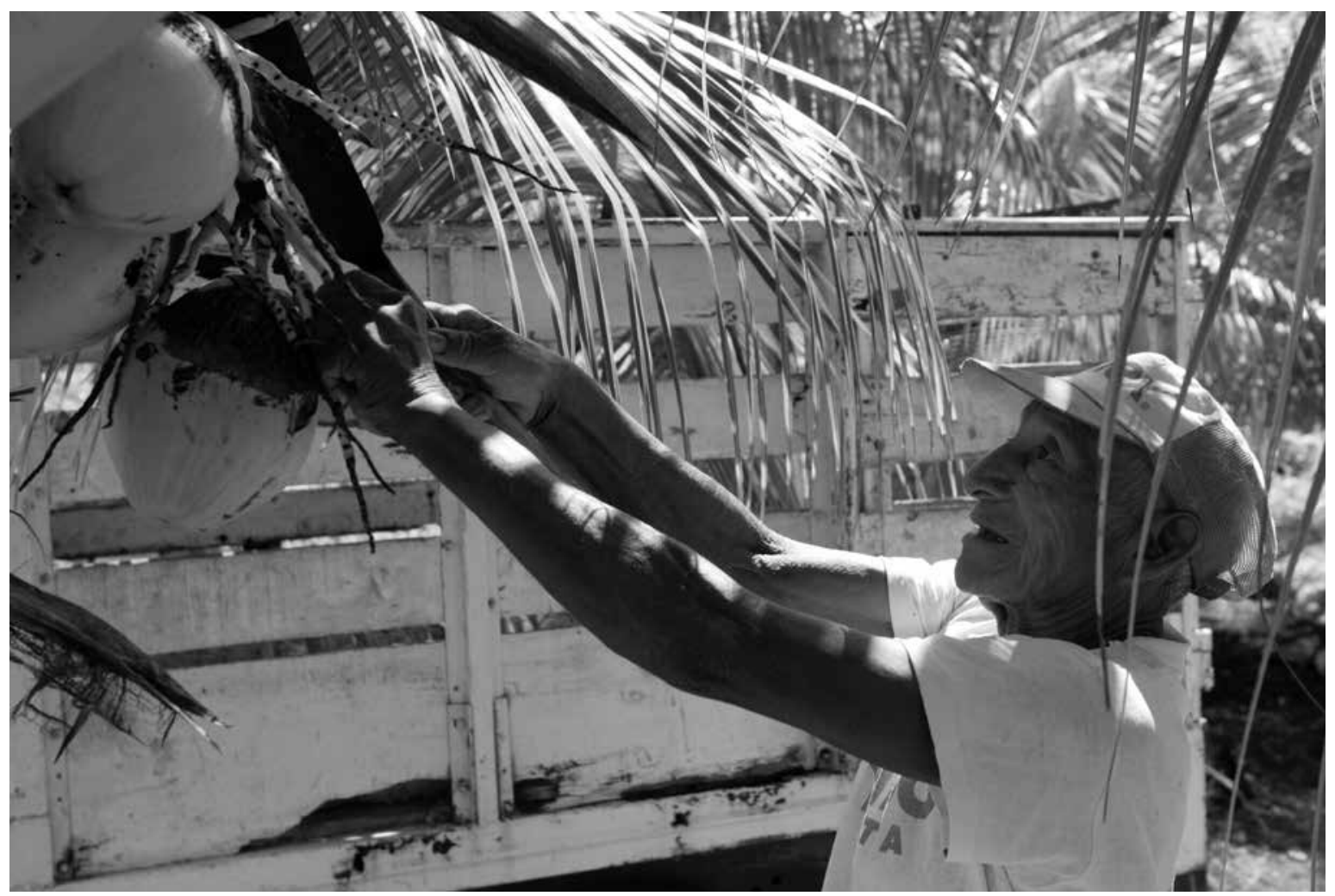

Octavio Hoyos Trabajador de la ex hacienda henequenera Oxtapacab. Mérida, Yucatán, México, 4 de junio de 2015.

de 1915, cuando recorrió las páginas de la Filosofía elemental, del pensador catalán Jaume Balmes. Con la convicción de quien halla su destino revelado, identificó en esas horas de lectura el origen exacto de su vocación filosófica. Pero más allá de la certeza con que distinguió ese instante inaugural, las dudas e imprecisiones impusieron su ley por encima de la endeble capacidad de evocación.

Cerrando los ojos, concentrándome sobre las imágenes emergentes en la memoria, me veo "por fuera" con precisa fidelidad: el mirador a la calle Campomanes del comedor de casa de mis abuelos; la butaca al lado del mirador; yo encogido en ella con el libro entre las manos... Pero cuando quiero, en un movimiento de film, agrandar ocupando la pantalla únicamente "por dentro" y leyendo, a través de los ojos, el libro, lo que veo se reduce a visiones y emociones posteriores, incluso en parte posiblemente tan sólo imaginarias, ficticias... ${ }^{20}$

Por efecto de las lagunas en el recuerdo y los artificios inherentes al ejercicio introspectivo, comprendió que su empresa comenzaba a desmoronarse. Al menos podía intuirlo ante la inoperancia del principio de identidad que sostenía el proyecto, aquel que estipula, por lo menos desde el siglo XIX, la posibilidad de conocerse a sí mismo a partir de una honesta indagación. Desde esa perspectiva, quien volviera la mirada hacia el interior y se observara con atención sería capaz de liberar los secretos encerrados en la 
bóveda de la conciencia. La transparencia del sujeto, en el sentido de constituir un objeto susceptible de la más completa e inmediata intelección, se presenta así como postulado de base en el tipo de discurso autobiográfico que Gaos había contemplado. ${ }^{21}$ Ahora bien, al confesarse inventor de una experiencia, por oposición a un mero portavoz, admitía que la realidad vivida, eje de su doctrina, se había oscurecido en el crisol de la memoria. La opacidad de su propio yo y de sus múltiples estratos se erigió así como el principal obstáculo hacia la inteligibilidad plena.

Las dificultades de tipo teórico y de orden práctico determinaron que el proyecto autobiográfico, como Gaos lo había entonces ideado, no pasara de algunos esbozos parciales y muy fragmentados - todos ellos conservados en su archivo-, y que con el transcurso del tiempo sus anotaciones se espaciaran. La inminencia del fracaso se encontró reflejada en un estado de ánimo cambiante, los instantes de entusiasmo se alternaban con otros, cada vez más frecuentes, de desaliento y obsesión autocrítica. La angustia y ansiedad diurnas lo perseguían también por las noches, como cuando entre sueños se veía cargando la cruz entre los ciriales o quemándose en la hoguera. ${ }^{22}$ Hacia 1941 se calificaba ya como un "filósofo raté". Algún tiempo después llegó a admitir que "el individuo en cuanto tal está condenado a no comprenderse". ${ }^{23}$ Las enseñanzas de Freud habían encontrado un lugar en su pensamiento y experiencia.

\section{El psicoanálisis en el horizonte contemporáneo de sentido}

Con la templanza que prestan los años, José Gaos se esforzó en su madurez por desenredar los hilos que se habían enlazado hasta conformar la trama contemporánea. "Historia de nuestra idea del mundo" fue el título que enmarcó ese inmenso tapiz, expuesto en una serie de lecciones que dictó entre
1966 y 1967 en El Colegio de México. En la medida en que habían incidido en los valores vigentes, los grandes pensadores y sus mayores aportaciones aparecieron en ese recuento, en el que se narra nada menos que la gesta intelectual del hombre moderno. Entre ese cúmulo de autores y nociones, no olvidó mencionar a Sigmund Freud, en tanto forjador de una antropología radical y de un nuevo sentido de la cultura. La progresiva difusión del psicoanálisis, su influencia en diversos movimientos artísticos y literarios, y el lugar acordado a los sueños no eran sino una muestra de su impronta en las concepciones efectivas. Igualmente revelador resultaba su influjo en la moral, "que va desde la abolición de las convenciones sociales que impedían hablar libre, públicamente, de lo sexual, hasta el reemplazo del tomarlo en una actitud religioso-moral por el tomarlo en una actitud psicológico-médica" (Gaos, 1994: 528). Por último, destacaba que hubiera desvelado la presencia de lo irracional y lo inconsciente en la razón y en la conciencia, gesto revolucionario que transformó el concepto mismo de la identidad y sus recursos.

Que esas transformaciones hicieron mella en su espíritu queda de manifiesto en el abandono del proyecto autobiográfico comenzado en la década de 1940. En 1958 había dado a la imprenta, eso sí, sus llamadas Confesiones profesionales, libro en el que expuso sus mayores ideas, su experiencia filosófica y los principales hitos de su trayectoria intelectual. Aunque se sitúa entre sus escritos mejor logrados, esta obra constituye, por su naturaleza, alcance y extensión, una pálida sombra de la empresa ambicionada hacía casi dos décadas. "Imposible ya

\footnotetext{
21 Gaos mismo habló de su experimento como de un proceso de "patentización", es decir, como si su injerencia se limitara a trasladar datos del inconsciente a la conciencia. AJG, 1, exp. 82, f. 16120.

22 AJG, 1, exp. 100, ff. 20130 y 20143.

23 AJG, 2, exp. 34, f. 35847; AJG, 1, exp. 12, f. 1578.
} 
prescindir de los conocimientos de la ciencia en el de sí mismo", señaló por aquellas fechas para de este modo dejar constancia de los efectos operados en sus ideas. ${ }^{24}$ Convertido en un enigma para sí, el hombre contemporáneo debería desde entonces recurrir al Otro —el biólogo, el médico, el psicoanalistapara dar razón de sus impulsos y temperamento. ${ }^{25}$ Una revolución se había verificado al descubrir que la identidad obedecía a normas invisibles, involuntarias e inescrutables para la propia conciencia.

A horcajadas entre dos modelos, la autobiografía de Gaos respondía a una lógica inaugurada en el siglo XVII con el Discurso del método, de René Descartes, y las interrogaciones en torno a las condiciones de toda intelección. ${ }^{26}$ El carácter convencional de su proyecto se disuelve, no obstante, al advertir el papel del psicoanálisis en sus exploraciones de la noche oscura del alma, así como en las nociones con las que bogó en esa penumbra. De ahí que tanto las claves que orientaron su introspección, como el destino que prestó a esos ejercicios resulten impensables antes de que comenzara el siglo Xx. El carácter históricamente situado de toda identidad queda así de manifiesto, y en primer lugar, las relaciones recíprocas entre lo que Norbert Elias denominó "las estructuras de la personalidad" y las "estructuras sociales". Esa solidaridad explica que unas y otras se condicionen e iluminen mutuamente: mientras que las transformaciones en el ámbito de lo íntimo y en la comprensión de sí sólo se tornen comprensibles a la luz de las determinaciones colectivas que las posibilitan y prestan sentido, la "organización espiritual" es indisociable de las relaciones y los hábitos, tanto físicos como mentales, que se van entretejiendo en una sociedad. ${ }^{27} \mathrm{~A}$ ello se debe que examinar la concepción del sujeto constituya una tarea insoslayable al momento de intentar reconstruir los contextos del pasado. La exigencia no decrece cuando la mirada se centra en una vida en concreto. Por el contrario, ¿qué otro género está a la vez más obligado y equipado para ocuparse de la génesis de una identidad que la biografía, en tanto estudio y testimonio de la historicidad humana?

AJG, 4, exp. 4, f. 62486, 4 de agosto de 1959.

25 Gaos estaba consciente de que estas ideas y su fuerza se circunscribían a un segmento limitado de la población. En alguna nota, contemporánea a la composición de Historia de nuestra idea del mundo, escribió admirado: "Qué libros históricamente revolucionarios, El Origen de las Especies, El Capital, o los de Marx, los de Freud. Ningún otro equiparable ni en la ciencia, ni en la filosofía, que no han revolucionado más que en sus propios dominios, herméticos a las masas". AJg, 1, exp. 117, f. 22907.

26 Así lo reconoció Gaos, al sostener que "autobiográficamente debo al Discurso del método antes que a nada la concepción 'confesional' de la filosofía. Más tarde supe que históricamente la debo a Montaigne”. AJG, 4, exp. 4, f. 62486, 4 de agosto de 1959.

27 En palabras de Elias: "Aquí ya no se trata solamente de las transformaciones de los 'conocimientos', de los cambios en las 'ideologías', en una palabra, de las alteraciones en los contenidos de la conciencia, sino que se trata de los cambios de todos los hábitos humanos, dentro de los cuales los contenidos de la conciencia, sobre todo los hábitos mentales, sólo constituyen una manifestación parcial, un sector aislado. Aquí se trata de las modificaciones de toda la organización espiritual en la totalidad de sus ámbitos, desde la orientación consciente del yo hasta la orientación completamente inconsciente de los instintos" (Elias, 2012: 587).

Bibliografía

Anzieu, Didier, 1978, El autoanálisis de Freud. El descubrimiento del psicoanálisis, 2 vols., Siglo xxı Editores, México.

Bödeker, Hans Erich, 2003, "Biographie. Annäherungen an den gegenwärtigen Forshungs- und Diskussionsstand”, en Hans Erich Bödeker (ed.), Biographie schreiben, Wallstein/Max-Planck-Institut für Geschichte, Gotinga, pp. 9-63.

Dosse, François, 2013, Paul Ricoeur. Los sentidos de una vida (1913-2005), Fondo de Cultura Económica, Buenos Aires. 
Eco, Umberto, 2009, El vértigo de las listas, Lumen, Barcelona.

Elias, Norbert, 2012, El proceso de la civilización. Investigaciones sociogenéticas y psicogenéticas, Fondo de Cultura Económica, México.

Gallo, Rubén, 2014, Freud en México: historia de un delirio, Fondo de Cultura Económica, México.

Gaos, José, 1994, Obras completas, t. xIv: Historia de nuestra idea del mundo, Universidad Nacional Autónoma de México, México.

Gaos, José y Francisco Larroyo, 2003, "Dos ideas de la filosofía (pro y contra la filosofía de la filosofía)”, en José Gaos, Obras completas, t. III: Ideas de la filosofía (1938-1950), Universidad Nacional Autónoma de México, México.

Gay, Peter, 2010, Freud: una vida de nuestro tiempo, Paidós, Barcelona.

Girard, Alain, 1996, “El diario como género literario”, en Revista de Occidente, núms. 182-183, pp. 31-38.

Goff, Jacques Le, 1993, "Les retours dans l'historiographie française actuelle”, en Carlos Barros (ed.), Historia a debate. Actas del congreso internacional "A historia a debate", Santiago de Compostela, del 7 al 11 de junio, pp. 157-165.

Loriga, Sabina, 2010, Le petit X. De la biographie à l'histoire, Seuil, París.

Menéndez Samará, Adolfo, 1940, "Reseña de José Gaos y Francisco Larroyo, Dos ideas de la filosofía”, en Letras de México, vol. II, núm. 15, 15 de marzo, p. 4.

Mier, Raymundo, 2008, "Norbert Elias, transfiguraciones de lo político: escritura, civilización e individuación”, en Valentina Torres Septién (coord.), El impacto de la cultura de lo escrito, Universidad Iberoamericana, México, pp. 131-160.

O’Gorman, Edmundo, 1945, "Cinco años de historia en México", en Filosofía y Letras, núm. 20, pp. 167-183.

Ortega y Gasset, José, 2004, Obras completas, t. I, Taurus, Madrid.

- 2005, Obras completas, t. III, Taurus, Madrid.

Ricoeur, Paul, 1965, De l'interprétation: Essai sur Freud, Seuil, París.

Saiz, Milagros y Dolores Saiz (coords.), 1996, Personajes para una historia de la psicología en España, Pirámide/Universitat Autònoma de Barcelona, Barcelona.

Sánchez Barranco-Ruiz, Antonio, 1996, “Ortega y Gasset y sus propuestas psicológicas”, en Milagros Saiz y Dolores Saiz (coords.), Personajes para una historia de la psicología en España, Pirámide/Universitat Autònoma de Barcelona, Barcelona, pp. 339-353.

Schoppa, R. Keith, 2004, "Culture and Context in Biographical Studies: The Case of China”, en Lloyd E. Ambrosius (ed.), Writing Biography. Historians \& Their Craft, University of Nebraska, Nebraska, pp. 27-52.

Torchia Estrada, Juan Carlos, 1992, "Correspondencia entre José Gaos y Francisco Romero”, en Revista de Filosofía y Teoría Política, núms. 28-29, pp. 159-194.

Valero Pie, Aurelia, 2012, José Gaos en México: una biografía intelectual, 1938-1969, tesis de doctorado en historia, El Colegio de México, México.

Vera Ferrándiz, Juan Antonio, 1996, “El porvenir de una ilusión: Ángel Garma y el psicoanálisis en España”, en Milagros Saiz y Dolores Saiz (coords.), Personajes para una historia de la psicología en España, Pirámide/Universitat Autònoma de Barcelona, Barcelona, pp. $423-445$.

\section{Archivos}

Archivo José Gaos (AJG), Instituto de Investigaciones Filosóficas, Universidad Nacional Autónoma de México. 